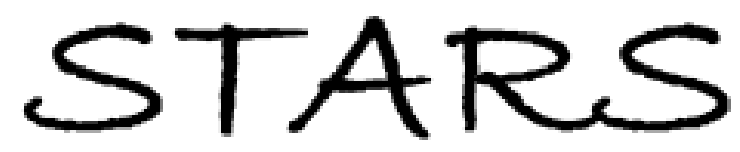

University of Central Florida

STARS

$1-1-2008$

\title{
Microring resonators fabricated by electron beam bleaching of chromophore doped polymers
}

\author{
Haishan Sun
}

Antao Chen

Benjamin C. Olbricht

Joshua A. Davies

Philip A. Sullivan

See next page for additional authors

Find similar works at: https://stars.library.ucf.edu/facultybib2000

University of Central Florida Libraries http://library.ucf.edu

This Article is brought to you for free and open access by the Faculty Bibliography at STARS. It has been accepted for inclusion in Faculty Bibliography 2000s by an authorized administrator of STARS. For more information, please contactSTARS@ucf.edu.

\section{Recommended Citation}

Sun, Haishan; Chen, Antao; Olbricht, Benjamin C.; Davies, Joshua A.; Sullivan, Philip A.; Liao, Yi; and Dalton, Larry R., "Microring resonators fabricated by electron beam bleaching of chromophore doped polymers" (2008). Faculty Bibliography 2000s. 1027.

https://stars.library.ucf.edu/facultybib2000/1027

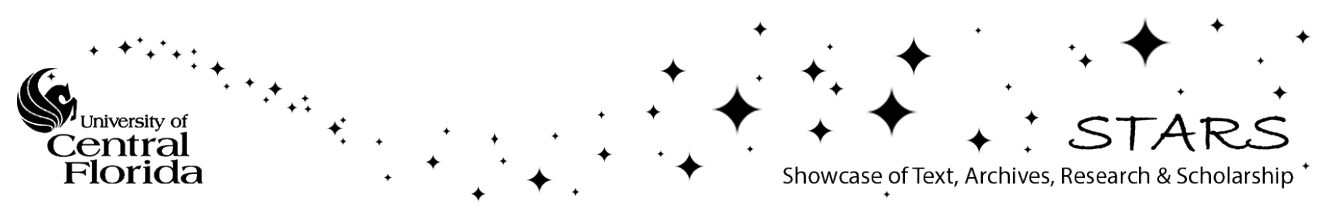


Authors

Haishan Sun, Antao Chen, Benjamin C. Olbricht, Joshua A. Davies, Philip A. Sullivan, Yi Liao, and Larry R. Dalton 


\section{Microring resonators fabricated by electron beam bleaching of chromophore doped polymers}

Cite as: Appl. Phys. Lett. 92, 193305 (2008); https://doi.org/10.1063/1.2926680

Submitted: 13 February 2008 . Accepted: 22 April 2008 . Published Online: 13 May 2008

Haishan Sun, Antao Chen, Benjamin C. Olbricht, Joshua A. Davies, Philip A. Sullivan, Yi Liao, and Larry R. Dalton

\section{Applied Physics Letters}

Mid-IR and THz frequency combs special collection 


\title{
Microring resonators fabricated by electron beam bleaching of chromophore doped polymers
}

\author{
Haishan Sun, ${ }^{1, a)}$ Antao Chen, ${ }^{1,2, b)}$ Benjamin C. Olbricht, ${ }^{3}$ Joshua A. Davies, ${ }^{3}$ \\ Philip A. Sullivan, ${ }^{3}$ Yi Liao, ${ }^{4}$ and Larry R. Dalton ${ }^{1,3, \mathrm{c})}$ \\ ${ }^{1}$ Department of Electrical Engineering, University of Washington, Seattle, Washington 98195, USA \\ ${ }^{2}$ Applied Physics Laboratory, University of Washington, Seattle, Washington 98105, USA \\ ${ }^{3}$ Department of Chemistry, University of Washington, Seattle, Washington 98195, USA \\ ${ }^{4}$ Department of Chemistry, University of Central Florida, Orlando, Florida 32816, USA
}

(Received 13 February 2008; accepted 22 April 2008; published online 13 May 2008)

\begin{abstract}
Decomposition of chromophore molecules under direct electron beam irradiation reduces the refractive index of chromophore containing polymers. The induced refractive index contrast between the exposed and unexposed regions is high enough for waveguide bends of small radius and thus microring resonator devices. This electron beam bleaching of chromophore-containing polymers provides a fabrication approach for nonlinear polymer optical waveguide devices. Fabrication of high quality microring resonators with critical feature size on the order of $100 \mathrm{~nm}$ was demonstrated with this technique in an electro-optic polymer that contains YL124 chromophores. (C) 2008 American Institute of Physics. [DOI: 10.1063/1.2926680]
\end{abstract}

Photonic integration can provide size, weight, and power reductions together with better performance analogous to electronic integration. Microring resonators are regarded as the promising building blocks because of their compactness and multifunctionality. ${ }^{1}$ For example, versatile functions of electro-optic (EO) modulation and switching, optical rectification, wavelength conversion, and all-optical switching can be realized by microring resonators made of organic nonlinear optical materials. ${ }^{2-4}$ Exponential increasing of the EO coefficients in a rate consistent with Moore's law and the intrinsic ultrafast response time of these materials could allow "lossless" conversion or even gain in the electricaloptical-electrical signal transduction process and terahertz bandwidths. ${ }^{5}$

Typical organic nonlinear optical materials are guesthost systems with optical polymers doped with organic chromophores. Systematic analysis and simulation of polymer microring resonators show that the fabrication of the submicron coupling gap with a precision of $<100 \mathrm{~nm}$ is critical to achieve high quality factor ( $Q$ factor) and sharp resonance. ${ }^{6}$ This high resolution can be achieved through electron beam lithography combined with reactive ion etching (RIE) or nanoimprinting. ${ }^{7}$ Traditional photolithography plus RIE have also been used to fabricate microring resonators with lateral coupling gaps greater than $1 \mu \mathrm{m}$ or vertical coupling gaps. ${ }^{2,8}$ However, many device designs require lateral coupling gaps smaller than $2 \mu \mathrm{m}$. For the chromophorecontaining polymers, high energy photons or electron irradiation can break bonds and decompose the organic chromophores. This decomposition bleaches out the color and reduces the index of refraction of the polymers. Previously, we have reported the fabrication of EO polymer microring resonators with photobleaching. ${ }^{9}$ In this letter, we recommend electron beam bleaching as an alternative approach for making chromophore-containing polymer microring resonators. Electron beam irradiation induced a refractive index

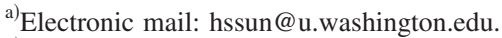

${ }^{b}$ Electronic mail: antaochen@apl.washington.edu.

${ }^{c)}$ Electronic mail: dalton@chem.washington.edu.
}

decrease of about 0.06 in chromophore-containing polymethyl methacrylate (PMMA) polymers, which is large enough for ring waveguides of small radius. Electron beam provides nanometer scale resolution, which could not be achieved with photobleaching. Without using expensive high resolution photomasks, different designs of microring resonator devices can be easily generated with computer assisted design and control systems. This is especially cost effective for device prototyping and design optimization. Electron beam bleaching is a single step process, which simplifies the device fabrication and reduces the sources of error from multiple fabrication steps. It also eliminates the use of wet chemicals that can dissolve the chromophore-containing polymer waveguides and greatly increase the propagation loss.

The chromophore used in this study was YL124. This chromophore has been used for optical rectification and EO modulation. ${ }^{3}$ When doped with binary chromophore organic glass materials, such as DR1-PMMA, an EO coefficient $r_{33}$ of more than $200 \mathrm{pm} / \mathrm{V}$ at $100 \mathrm{~V} / \mu \mathrm{m}$ electric poling field has been reported. ${ }^{10}$ YL124 was doped into PMMA host polymer with a loading density of $20 \%$. The PMMA polymer has a molecular weight of 950000 and PMMA of this molecular weight is used as high resolution electron beam resist. The solid mixture of PMMA and chromophore was dissolved in chlorobenzene and the total solid content is about $7.75 \%$. The solution was spin coated at a spin rate of $3000 \mathrm{rpm} / \mathrm{min}$ onto a Si substrate with UV curable polymer UV16 (Master Bond, Inc.) precoated and formed an EO polymer film of about $2 \mu \mathrm{m}$. The UV16 layer served as a lower cladding and it had a thickness of about $3.6 \mu \mathrm{m}$. The EO polymer film was then baked in a vacuum oven at $65{ }^{\circ} \mathrm{C}$ for two days to completely remove residue solvent.

The EO polymer film was patterned with FEI Sirion scanning electron microscope equipped with a nanometer pattern generation system (NPGS). Accelerating voltage of $30 \mathrm{kV}$ and beam current of $5 \mathrm{nA}$ were used to have a shorter writing time. We first made waveguides with $90^{\circ}$ bends to test the appropriate exposure dose. Bent waveguides with $500 \mu \mathrm{m}$ bending radius and $5 \mu \mathrm{m}$ width were exposed with 


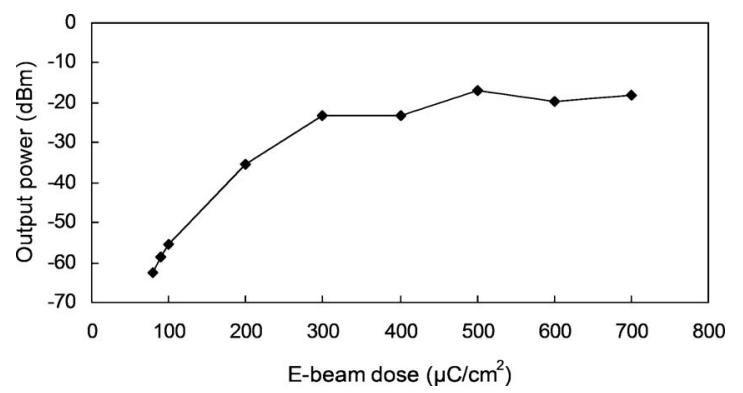

FIG. 1. Measured output optical powers of the electron beam patterned bent waveguides as functions of the electron beam doses.

different electron beam doses and the propagation loss of these waveguides was measured. The strip areas on both sides of the waveguide were exposed to electron beam irradiation and functions such as cladding to provide lateral confinement for the waveguide. Bleaching of the dark green color EO polymers under electron beam irradiation is clearly visible under an optical microscope. Total width including waveguides and the two exposed strips is $50 \mu \mathrm{m}$. A broadband erbium amplified spontaneous emission source with wavelength distribution of around $1520-1560 \mathrm{~nm}$ was coupled to the waveguide through a polarizer to launch the transverse magnetic (TM) mode in a polarization maintaining (PM) optical fiber that is end coupled to the input end of the waveguide. The output of the waveguide was lens coupled onto a photodetector of an optical power meter (Agilent $81623 \mathrm{~B}$ ). Figure 1 shows that output light power increases with the electron beam dose. This trend is consistent with the refractive index change of the EO polymer after e-beam bleaching. The change saturates when the electron beam dose is above $500 \mu \mathrm{C} / \mathrm{cm}^{2}$.

The color of the e-beam exposed area changed from dark blue to very light blue. The characteristic absorption peak of the chromophore also diminished (Fig. 2). Refractive index and thickness changes of the EO polymer film under electron beam irradiation were measured with the prism coupling technology (Metricon 2010). A $2 \mathrm{~mm}$ diameter circular area on the EO polymer film was exposed with $700 \mu \mathrm{C} / \mathrm{cm}^{2}$ electron beam. More than three spots on both the electron beam exposed and unexposed regions were measured. The average refractive index at the wavelength of $1550 \mathrm{~nm}$ for the unexposed area was 1.5581 and that of the exposed area was 1.4961. The standard deviations were smaller than 0.0002 and confirmed the self-consistency and validity of the measurements. The index decrease induced by the electron beam

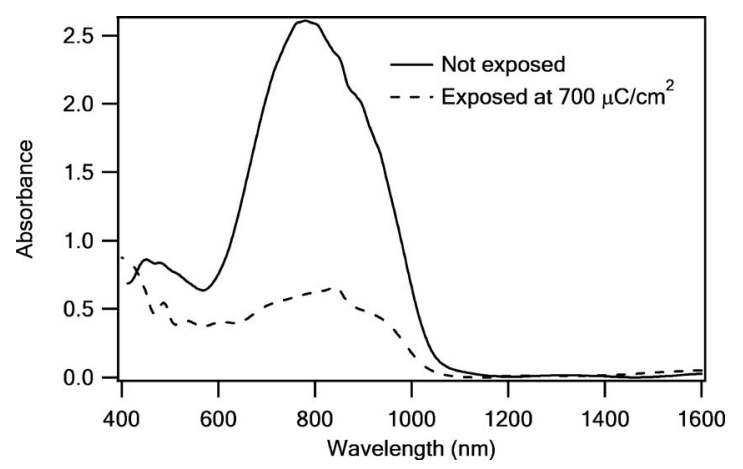

FIG. 2. Absorption spectra of an YL124/PMMA thin film before and after electron beam bleaching.

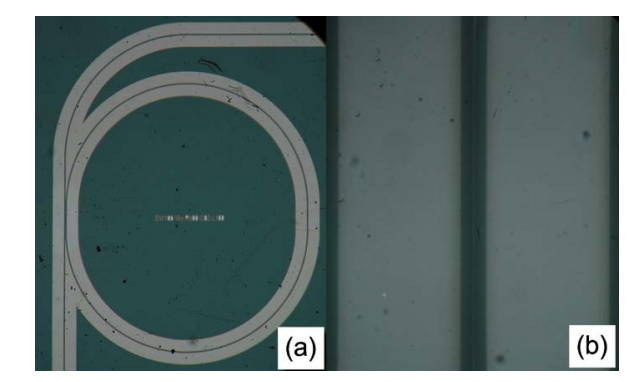

FIG. 3. (Color online) Microring resonators fabricated with the electron beam bleaching method. (a) Microscopic picture of the device. The lighter areas are bleached by the electron beam irradiation. (b) Close microscopic view of the coupling region. A $200 \mathrm{~nm}$ coupling gap was clearly resolved.

irradiation was 0.0620 and about $4 \%$ of the original value. This result contrasts to the refractive index increase in pure PMMA induced by the electron beam irradiation, ${ }^{11}$ which indicates that the change of the refractive index should be mainly attributed to the decomposition of organic chromophore under high energy electron beam irradiation. This can be further verified with the refractive index of the exposed area, which is only a little bigger than the refractive index of the pure PMMA (1.488). The film thickness decreased by $0.12 \mu \mathrm{m}$ and $4.6 \%$ of the original value. This could be due to the escaping of volatile small species decomposed from the chromophore out of the EO polymer. The same phenomena were also observed in photobleaching of EO polymers.

Electron beam bleaching of the EO polymer was used to make microring resonators of the basic structure with a single ring resonator coupled to one bus waveguide. The EO polymer and its thickness were the same as above and the electron beam dose was also $700 \mu \mathrm{C} / \mathrm{cm}^{2}$. Si substrate with a $5 \mu \mathrm{m}$ thermal silicon dioxide layer was used. The silicon dioxide was used as the lower cladding and its refractive index is 1.46. Microring resonators of different designs were fabricated and the transmission wavelength spectra were measured. Individual devices were cleaved from the wafer and the two ports of the bus waveguides were end coupled to the input and output optical fibers. Output from a tunable laser source (Santec TSL-210) was connected to an Agilent polarization controller. TM or TE polarized light was then end coupled to the input port of the microring resonators through the PM fiber. An Agilent 81623B optical power meter was used to measure the optical power coupled from the output port. Scanning of the input light wavelength and acquiring of the output optical power were controlled by a computer program.

The highest resonance extinction ratio was achieved in a microring resonator with a waveguide width of $5 \mu \mathrm{m}$, total width including waveguides and the two exposed strips of $100 \mu \mathrm{m}$, and coupling gap size of $200 \mathrm{~nm}$. The ring resonator had a racetrack shape with circular sections of $500 \mu \mathrm{m}$ radius and straight coupling section of $100 \mu \mathrm{m}$ in length (Fig. 3). The extinction ratio was $12 \mathrm{~dB}$ for TE polarization and $9 \mathrm{~dB}$ for TM polarization. No higher resonance modes presented in both of the resonance spectra. This confirmed the single mode operation of the ring waveguide. By fine tuning the gap size with a resolution smaller than $100 \mathrm{~nm}$ and the coupling length, the critical coupling condition can be further approached and a higher extinction ratio is possible. From the measurements of different microring resona- 


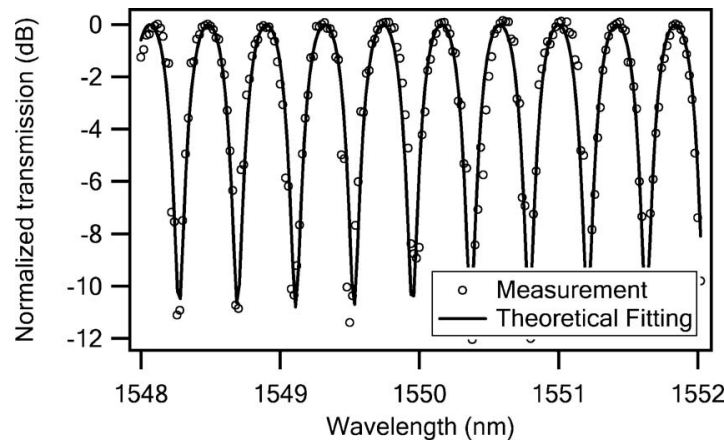

FIG. 4. Normalized transmission spectrum of a microring resonator. The input light is TE polarized.

tor designs, the two exposed strips outside the waveguide can be less than $7.5 \mu \mathrm{m}$ wide, which consumes a much shorter electron beam writing time. Ring radius of $300 \mu \mathrm{m}$ or smaller is also possible. The resonance spectrum of the TE polarization was fitted to the theoretical transfer function (Fig. 4). ${ }^{12}$ The fitted internal circulation attenuation factor $\alpha$ is 0.55 and coupling attenuation factor $t$ is 0.36 . From these fitting results, $Q$ factor was calculated to be 9450 , ring waveguide propagation loss is $16 \mathrm{~dB} / \mathrm{cm}$, and finesse is 2.7 . The main loss mechanism may be the scattering at the waveguide edges because NPGS allows limited vertices along the arc polygons, which could reduce the edge smoothness. By splitting the polygons to smaller sections or using a better electron beam writing system, the waveguide loss could be reduced and $Q$ factor could be even higher.

In conclusion, decomposition of chromophore molecules under electron beam irradiation reduces the refractive index of the chromophore containing polymers. The refractive index change by electron beam bleaching is large enough for small waveguide bending and thus microring resonator devices. The electron beam bleaching techniques were used to directly write microring resonators of high extinction ratio and quality factor. Electron beam bleaching is a single step, maskless, resistless direct writing process. Coupling gap of $100 \mathrm{~nm}$ or smaller can be resolved with a standard scanning electron microscopy with an electron beam lithography pat- tern generator. The fabrication process avoids organic solvents that might attack the chromophore containing polymers and is compatible to a broader material system. Microring resonators made of unpoled chromophore containing polymers can be used as passive wavelength filters or sensors, e.g., chemical sensors for trace dinitrotoluene explosive detection. ${ }^{13}$ After the chromophore molecules are properly aligned with the poling electric field, active microring resonator devices with the functions of EO modulation, switching, and tunable filtering could be realized.

This work is supported by NSF under Grant No. ECS0437920 and NSF Center on Materials and Devices for Information Technology Research (CMDITR) under Grant No. DMR-0120967. The work was conducted at the Nanotech User Facility at the University of Washington, a member of the National Nanotechnology Infrastructure Network (NNIN) supported by NSF. The authors thank Steven Hau and $\mathrm{Su}$ Huang for their help with the refractive index measurement.

${ }^{1}$ B. E. Little and S. T. Chu, Opt. Photonics News 11, 24 (2000).

${ }^{2}$ P. Rabiei, W. H. Steier, C. Zhang, and L. R. Dalton, J. Lightwave Technol. 20, 1968 (2002).

${ }^{3}$ T. Baehr-Jones, M. Hochberg, G. Wang, R. Lawson, Y. Liao, P. A. Sullivan, L. Dalton, A. K.-Y. Jen, and A. Scherer, Opt. Express 13, 5216 (2005).

${ }^{4}$ M. Hochberg, T. Baehr-Jones, G. Wang, M. Shearn, K. Harvard, J. Luo, B. Chen, Z. Shi, R. Lawson, P. Sullivan, A. K.-Y. Jen, L. Dalton, and A. Scherer, Nat. Mater. 5, 703 (2006).

${ }^{5}$ L. Dalton, SPIE Newsroom (http://spie.org/x16998.xml?highlight $=\mathrm{x} 2414)$.

${ }^{6} \mathrm{H}$. Sun, L. Dalton, and A. Chen, Digest of the IEEE LEOS Summer Topical Meetings (IEEE, Portland, 2007), p. 217.

${ }^{7}$ Y. Huang, G. T. Paloczi, A. Yariv, C. Zhang, and L. Dalton, J. Phys. Chem. 108, 8606 (2004).

${ }^{8}$ H. Tazawa, Y.-H. Kuo, I. Dunayevskiy, J. Luo, A. K.-Y. Jen, H. R. Fetterman, and W. H. Steier, J. Lightwave Technol. 24, 3514 (2006).

${ }^{9}$ J. Zhou, A. Pyayt, L. R. Dalton, J. Luo, A. K.-Y. Jen, and A. Chen, IEEE Photonics Technol. Lett. 18, 2221 (2006).

${ }^{10}$ L. Dalton, SPIE Newsroom (http://spie.org/x17559.xml).

${ }^{11}$ H. Kotani, M. Kawabe, and S. Namba, Jpn. J. Appl. Phys. 18, 279 (1979).

${ }^{12}$ A. Yariv, Electron. Lett. 36, 321 (2000).

${ }^{13}$ A. Pyayt, X. Zhang, J. Luo, A. Jen, L. Dalton, and A. Chen, Proc. SPIE 6556, 65561D (2007) 